\title{
Development of a new approach for deterministic supply chain network design
}

\begin{abstract}
This paper proposes a mixed integer linear programming model and solution algorithm for solving supply chain network design problems in deterministic, multi-commodity, singleperiod contexts. The strategic level of supply chain planning and tactical level planning of supply chain are aggregated to propose an integrated model. The model integrates location and capacity choices for suppliers, plants and warehouses selection, product range assignment and production flows. The open-or-close decisions for the facilities are binary decision variables and the production and transportation flow decisions are continuous decision variables. Consequently, this problem is a binary mixed integer linear programming problem. In this paper, a modified version of Benders' decomposition is proposed to solve the model. The most difficulty associated with the Benders' decomposition is the solution of master problem, as in many reallife problems the model will be NP-hard and very time consuming. In the proposed procedure, the master problem will be developed using the surrogate constraints. We show that the main constraints of the master problem can be replaced by the strongest surrogate constraint. The generated problem with the strongest surrogate constraint is a valid relaxation of the main problem. Furthermore, a near-optimal initial solution is generated for a reduction in the number of iterations.
\end{abstract}

Keyword: Facilities planning and design, Supply chain network design, Combinatorial optimization, Mixed integer linear programming, Benders' decomposition, Surrogate constraints 